\title{
RESEARCH ON SIMILARITY VALUES RELEVANT FOR FUZZY MATRIX CONSISTENCY CHECK
}

\author{
Zedina Lavic, Nedzad Dukic, Mugdim Pasic \& Branko Vucijak
}
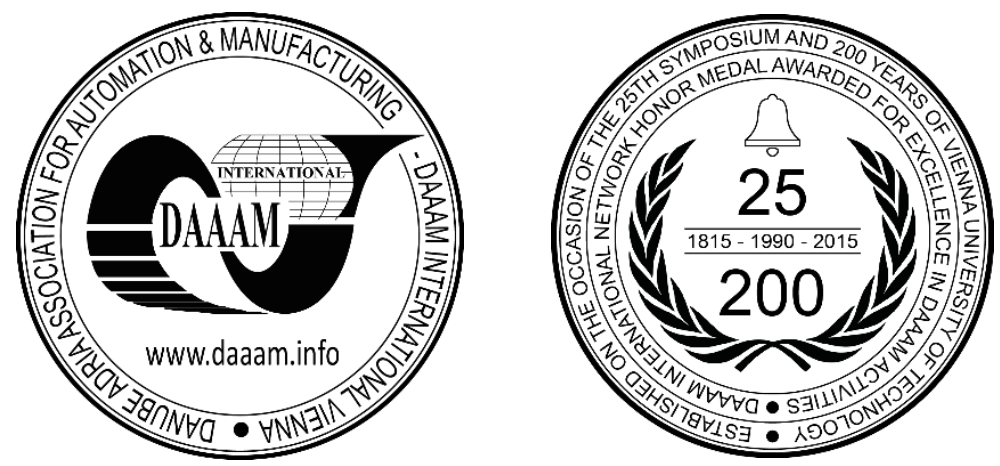

This Publication has to be referred as: Lavic, Z[edina]; Dukic, N[edzad]; Pasic, M[ugdim] \& Vucijak, B[ranko] (2016). Research on Similarity Values Relevant for Fuzzy Matrix Consistency Check, Proceedings of the 27th DAAAM International Symposium, pp.0645-0649, B. Katalinic (Ed.), Published by DAAAM International, ISBN 978-3-90273408-2, ISSN 1726-9679, Vienna, Austria

DOI: $10.2507 / 27$ th.daaam.proceedings.093

\begin{abstract}
There is a problem of inherent inconsistency when forming pairwise comparison matrices in both classical and fuzzy Analytic Hierarchy Process. The smallest decision making element of fuzzy Analytic Hierarchy Process is square, positive, fuzzy reciprocal matrix $3 \times 3$, and this paper focus to the research on fuzzy numbers' similarities regarding the consistency check of fuzzy matrix $3 \times 3$. The research is performed using Java application created specifically for this purpose. The application, for the selected fuzzy scale, forms all $3 \times 3$ matrices, for each matrix calculates all similarities values relevant for consistency check, average similarity and determines the minimal similarity value. The application calculates CR (consistency ratio) values for corresponding classical matrices. Research results are presented and future research suggested.
\end{abstract}

Keywords: Pairwise Comparison Matrix; Consistency; Fuzzy Numbers, Fuzzy Scale; Similarity Measure; Fuzzy Analytic Hierarchy Process

\section{Introduction}

Numerous different methods are developed to solve multicriteria decision making problems, mostly related to the strict numerical valuation of selected criteria - examples of papers using such methods written by the authors relate to multicriteria evaluation of supervisory boards[1], selection of buildings' insulation [2] or selection of best alternative for highway tunnel doors [3].

One of frequently used multicriteria decision making aid methods is Analytic Hierarchy Process (classical, but also fuzzy). Limited research is done so far regarding evaluation of consistency in forming the pairwise comparison matrices, with few papers available dealing with the issue of consistency of fuzzy pairwise comparison matrices (examples are [4, $5,6,7])$. According to the work of Buckley [8], fuzzy pairwise comparison matrix $\tilde{A}=\left\{\tilde{a}_{i j}\right\}$ is consistent if $\tilde{a}_{i k} \otimes \tilde{a}_{k j} \approx$ $\tilde{a}_{i j}$ where $i, j, k=1,2, \ldots, n$, and where " $\otimes$ " represents multiplication of fuzzy numbers, while " $\approx$ " means "fuzzy equal" [9].

The concept of fuzzy equality has the same meaning as the notion of similarity, since the similarity in essence is a generalization of the concept of equivalence [10]. Similarity is a relation which is an extension to the concept of functions 
belonging to the set of elements, where, abandoning the idea of exact equality, the elements of the domain are considered to have different degrees of similarity [11].

The application of fuzzy numbers in resolving problems requires the application of different similarity measures, calculating the degree of similarity between two fuzzy number; similarity measures appropriate for solving one type of problem often are not appropriate for solving the other problem or type of problems. Therefore, various similarity measures of fuzzy numbers exist [11].. The similarity measure of triangular fuzzy numbers proposed by Chen and Lin ([7]) is based on distances between the midpoints and the appropriate boundary points of the support interval: if $A=$ $\left(a^{l}, a^{m}, a^{u}\right)$ and $B=\left(b^{l}, b^{m}, b^{u}\right)$ are triangular fuzzy numbers, similarity can be calculated with

$$
S_{M B}(A, B)=1-\frac{\left|a^{l}-b^{l}\right|+\left|a^{m}-b^{m}\right|+\left|a^{u}-b^{u}\right|}{3}
$$

Hsieh and Chen have presented the utility value concept of triangular fuzzy numbers, and based on these values have also defined the similarity [9]:

$$
S_{U V}(A, B)=\frac{U_{U V}(A) x U_{U V}(B)}{\max \left(\left(U_{U V}(A)\right)^{2},\left(U_{U V}(B)\right)^{2}\right)} .
$$

The similarity measure is function fulfilling the following two conditions [9]:

- Reflexivity, i. e. $S(A, A)=1$ and

- Symmetry, i.e. $S(A, B)=S(B, A)$.

Similarity measure based on the distance is used in this paper (as defined in [9]):

$$
S(A, B)=\frac{1}{1+d(A, B)}
$$

where $d(A, B)$ is a measure of the distance defined with:

$$
d(A, B)=\left|a^{l}-b^{l}\right|+\left|a^{m}-b^{m}\right|+\left|a^{u}-b^{u}\right|,
$$

so the similarity measure used is

$$
S(A, B)=\frac{1}{1+\left|a^{l}-b^{l}\right|+\left|a^{m}-b^{m}\right|+\left|a^{u}-b^{u}\right|} .
$$

This similarity measure fulfils the conditions of reflexivity and symmetry.

\section{Research}

Pairwise comparison matrices of the fuzzy Analytic Hierarchy Process (fAHP) based decision making models are positive and reciprocal. The smallest matrix in fAHP is $3 \times 3$ pairwise comparison matrix, square, positive and reciprocal:

$$
\left[\begin{array}{ccc}
(111) & a_{12} & a_{13} \\
1 / a_{12} & (111) & a_{23} \\
1 / a_{13} & 1 / a_{23} & (111)
\end{array}\right]
$$

The elements $a_{i j}(i, j=1,2,3)$ are obtained using appropriate fuzzification of scale given by Saaty [13]. Original Saaty's scale and fuzzified Saaty's scale are presented with Tables $1 \& 2$ respectively.

\begin{tabular}{|c|c|c|}
\hline Definition & $\begin{array}{c}\text { Importance } \\
\text { intensity }\end{array}$ & Reciprocals \\
\hline Equal importance & 1 & 1 \\
\hline Moderate importance & 3 & $1 / 3$ \\
\hline Strong importance & 5 & $1 / 5$ \\
\hline Very strong importance & 7 & $1 / 7$ \\
\hline Extreme importance & 9 & $1 / 9$ \\
\hline Intermediate values & $2,4,6$ and 8 & $1 / 2,1 / 4,1 / 6$ and $1 / 8$ \\
\hline
\end{tabular}

Table 1. Scale given by Saaty 


\begin{tabular}{|c|c|c|}
\hline Definition & Importance intensity & Reciprocals \\
\hline Equal importance & $(1,1,1)$ & $(1,1,1)$ \\
\hline Moderate importance & $(2,3,4)$ & $(1 / 4,1 / 3,1 / 2)$ \\
\hline Strong importance & $(4,5,6)$ & $(1 / 6,1 / 5,1 / 4)$ \\
\hline Very strong importance & $(6,7,8)$ & $(1 / 9,1 / 9,1 / 9)$ \\
\hline Extreme importance & $(9,9,9)$ & $(1 / 3,1 / 2,1),(1 / 5,1 / 4,1 / 3),(1 / 7$, \\
\hline \multirow{2}{*}{ Intermediate values } & $(1,2,3),(3,4,5),(5,6,7)$ and & $1 / 6,1 / 5)$ and $(1 / 9,1 / 8,1 / 7)$ \\
\hline
\end{tabular}

Table 2. Fuzzified Saaty's scale

In order to explore relationship between the similarity measures value and consistency of fuzzy matrices, Java application is created and used. The application creates $17^{n(n-1) / 2}$ matrices, where $n$ is dimension of the particular matrix explored. For each created matrix application calculates similarity of fuzzy numbers $\tilde{a}_{i k} \otimes \tilde{a}_{k j}$ and $\tilde{a}_{i j}$, for every $i, j, k=1,2, \ldots, n$. After that, for each matrix the application finds minimum, calculates the average similarity and forms MS Excel file where all the fuzzy matrices with the corresponding minimum and average similarities are given. The application also calculates and presents CR value (consistency ratio) [13] for classical matrices derived from the fuzzy matrices. Matrices are formed using classical matrices' elements $a_{i j}$ representing the midpoint of the fuzzy numbers interval $\tilde{a}_{i j}=\left(a_{i j}^{l}, a_{i j}^{m}, a_{i j}^{u}\right)$. Thus, the elements of the classical matrix are $a_{i j}=a_{i j}^{m}$ for all $i, j=1,2, \ldots, n$. Similarity values (minimal and average) and CR values for fuzzy and classical matrices $3 \times 3$ respectively are explored. It should be noted that these matrices, as the corresponding values of displayed indicators, would be different if fuzzy scales would be different than those presented in the Table 2 .

\section{Results and Discussion}

For the case of selected fuzzy scale, all the values of minimal similarity of matrices $3 \times 3$ are rounded to 4 decimal places and they belong to the interval $[0.0041,1]$. There are total of 162 minimal similarity values, with the smallest popup value of 0.0041 , for the selected fuzzy scale. Analysis of the output Excel file, containing all matrices so as corresponding minimal and average similarities and CR values of correspondent classic matrices, is performed. It was found that for the fuzzy matrices whose minimal similarities belong to the interval [0.0041,0.0294] all correspondent classical matrices have CR values greater than $10 \%$, what means that they are not consistent. The total number of these matrices is 3576 and indicates the level of the problem of inconsistency check with matrices of dimension three. The total number of different minimal similarities within the range [0.0041,0.0294] is 122. For these matrices, the lowest average similarity is 0.6577 , the largest average similarity is 0.8297 , and the smallest CR is $10.1587 \%$. The focus of further analysis of the minimal similarities values is at the values belonging to the interval $[0.0303,1]$. The values for these similarities (a total of 40 values) are presented with Table 3.

\begin{tabular}{|c|c|c|c|c|c|c|c|}
\hline $\begin{array}{c}\text { Ordinal } \\
\text { Number }\end{array}$ & $\begin{array}{c}\text { Minimal } \\
\text { Similarity }\end{array}$ & $\begin{array}{c}\text { Ordinal } \\
\text { Number }\end{array}$ & $\begin{array}{c}\text { Minimal } \\
\text { Similarity }\end{array}$ & $\begin{array}{c}\text { Ordinal } \\
\text { Number }\end{array}$ & $\begin{array}{c}\text { Minimal } \\
\text { Similarity }\end{array}$ & $\begin{array}{c}\text { Ordinal } \\
\text { Number }\end{array}$ & $\begin{array}{c}\text { Minimal } \\
\text { Similarity }\end{array}$ \\
\hline 1. & 0.0303 & 11. & 0.0417 & 21. & 0.0667 & 31. & 0.2069 \\
\hline 2. & 0.0321 & 12. & 0.0435 & 22. & 0.0714 & 32. & 0.2500 \\
\hline 3. & 0.0323 & 13. & 0.0455 & 23. & 0.0759 & 33. & 0.2727 \\
\hline 4. & 0.0333 & 14. & 0.0476 & 24. & 0.0769 & 34. & 0.4000 \\
\hline 5. & 0.0346 & 15. & 0.0500 & 25. & 0.0833 & 35. & 0.4839 \\
\hline 6. & 0.0355 & 16. & 0.0522 & 26. & 0.0909 & 36. & 0.5455 \\
\hline 7. & 0.0357 & 17. & 0.0526 & 27. & 0.1000 & 37. & 0.5932 \\
\hline 8. & 0.0370 & 18. & 0.0556 & 28. & 0.1111 & 38. & 0.6316 \\
\hline 9. & 0.0397 & 19. & 0.0588 & 29. & 0.125 & 39. & 0.6632 \\
\hline 10. & 0.0400 & 20. & 0.0625 & 30. & 0.1429 & 40. & 1 \\
\hline
\end{tabular}

Table 3. Values of the minimal similarities from the interval [0.0303 to 1] 
Analysis of the entire range of minimal values of similarities [0.0041. 1] has shown that:

- For the minimal similarities lower than 0.0303 there is no consistent classical matrix.

- For some minimal similarities within the range $(0.0303,0.1429)$ there are corresponding average similarities for which both consistent and inconsistent classical matrices exist.

- For some minimal similarities within the range $(0.0303,0.1]$ there are corresponding average similarities for which classical matrices are always consistent (Table 4).

- When the minimal similarities are higher than 0.1 (except if the minimal similarity is 0.1429 and corresponding average similarity is 0.7873 , when CR of classical matrix is $12.7714 \%$ ). classical matrices are consistent.,

- For minimal similarities higher than 0.1429 all the classic matrices are consistent.

\begin{tabular}{|c|c|}
\hline Minimal Similarity & Average Similarity \\
\hline 0.0333 & 0.7919 \\
\hline \multirow{2}{*}{0.0357} & 0.8154 \\
\hline & 0.796 \\
\hline \multirow{3}{*}{0.037} & 0.7545 \\
\hline & 0.7627 \\
\hline & 0.7641 \\
\hline \multirow{4}{*}{0.0417} & 0.7583 \\
\hline & 0.7652 \\
\hline & 0.7962 \\
\hline & 0.7969 \\
\hline \multirow{2}{*}{0.0435} & 0.7673 \\
\hline & 0.7985 \\
\hline \multirow{2}{*}{0.0476} & 0.7494 \\
\hline & 0.7513 \\
\hline \multirow{2}{*}{0.05} & 0.7621 \\
\hline & 0.7984 \\
\hline 0.0526 & 0.7731 \\
\hline \multirow{4}{*}{0.0556} & 0.7245 \\
\hline & 0.7425 \\
\hline & 0.7637 \\
\hline & 0.8135 \\
\hline \multirow{2}{*}{0.0588} & 0.7559 \\
\hline & 0.7766 \\
\hline \multirow{2}{*}{0.0625} & 0.7699 \\
\hline & 0.8017 \\
\hline 0.0667 & 0.7312 \\
\hline
\end{tabular}

\begin{tabular}{|c|c|}
\hline Minimal Similarity & Average Similarity \\
\hline & 0.7459 \\
\hline & 0.7762 \\
\hline \multirow{5}{*}{0.0714} & 0.7316 \\
\hline & 0.7485 \\
\hline & 0.7647 \\
\hline & 0.7658 \\
\hline & 0.7999 \\
\hline \multirow{4}{*}{0.0769} & 0.7677 \\
\hline & 0.7888 \\
\hline & 0.7966 \\
\hline & 0.8338 \\
\hline \multirow{3}{*}{0.0833} & 0.7159 \\
\hline & 0.7767 \\
\hline & 0.7992 \\
\hline \multirow{4}{*}{0.0909} & 0.7105 \\
\hline & 0.7403 \\
\hline & 0.7492 \\
\hline & 0.7872 \\
\hline \multirow{7}{*}{0.1} & 0.7126 \\
\hline & 0.7586 \\
\hline & 0.7734 \\
\hline & 0.7808 \\
\hline & 0.8015 \\
\hline & 0.8166 \\
\hline & 0.8509 \\
\hline
\end{tabular}

Table 4. Minimal and average similarities in the range $(0.0303$ to 0.1$]$. CR $<10 \%$

\section{Conclusion}

Both for classical and for fuzzy Analytic Hierarchy Process, a problem of inconsistency of decision maker appears when forming the pairwise comparison matrices and this paper focuses on resolving it. Appropriate fuzzy scale, obtained by fuzzification of Saaty's scale, is used in this paper. Research on similarities of fuzzy numbers, relevant in terms of consistency check, resulted with the conclusion that the inconsistency issues appear even with matrices of smaller dimensions. For the case of selected fuzzy scale, all the values of minimal similarity of matrices $3 \times 3$, rounded to 4 decimal places, belong to the interval $[0.0041,1]$. It is found that for the fuzzy matrices whose minimal similarities are within the interval [0.0041, 0.0294] all correspondent classical matrices have CR values greater than 10\%, what means they are not consistent. Total number of these matrices is 3576 (total number of fuzzy matrices is 4913). For some minimal similarities within the range $(0.0303,0.1]$ there are corresponding average similarities for which classical matrices are always consistent. For minimal similarities higher than 0.1 (except if the minimal similarity is 0.1429 and corresponding average similarity is 0.7873 , when CR of classical matrix is $12.7714 \%$ ). classical matrices are consistent, and for minimal similarities higher than 0.1429 all the related classic matrices are consistent.

Since the matrix $3 \times 3$ is the basic element in fuzzy (and classical) Analytic Hierarchy Process, consistency check of fuzzy matrices of dimensions larger than 3 is feasible using decomposition of larger matrices into the matrices $3 \times 3$, what will be the subject of the further research. 


\section{References}

[1] Karic E., Vucijak B., Pasic M., Bijelonja I. (2011): „Multi-Criteria Evaluation of Supervisory Board Effectiveness“, Proceedings of the $22^{\text {nd }}$ DAAAM International Symposium on Intelligent Manufacturing and Automation, Volume 22, Vienna, 23-26 November 2011, $251-252$

[2] Civic A. and Vucijak B. (2013): „Multi-Criteria Optimization of Insulation Options for Warmth of Buildings to Increase Energy Efficiency“, $24^{\text {th }}$ DAAAM International Symposium on Intelligent Manufacturing and Automation, Zadar, 23-26 October 2013, Procedia Engineering 69 ( 2014 ) 911 - 920

[3] Vucijak B., Pasic. M., Zorlak A. (2015): „Use of Multi-Criteria Decision Aid Methods for Selection of the Best Alternative for the Highway Tunnel Doors “, $25^{\text {th }}$ DAAAM International Symposium on Intelligent Manufacturing and Automation, Zadar, 23-26 October 2013, Procedia Engineering 100 ( 2015 ) 656 - 665

[4] Ramik, J., Vlach, M. (2013). Measuring consistency and inconsistency of pair comparison systems, Kybernetika 49(3): 465-486 (2013)

[5] Ramik, J. (2014). Incomplete fuzzy preference matrix and its application to ranking of alternatives, Intelligent Systems 29, 8 (2014), 787-806

[6] Ramik, J., Korviny, P. (2010). Inconsistency of pair-wise comparison matrix with fuzzy elements based on geometric mean, Fuzzy Sets and Systems 161(11): 1604-1613 (2010)

[7] Ramik, J. (2009). Consistency of Pair-wise Comparison Matrix with Fuzzy Elements. IFSA/EUSFLAT Conf. 2009: 98-101

[8] Buckley, J. J. (1985), Fuzzy hierarchical analysis. Fuzzy Sets and Systems, 17(2), 233-247.

[9] Javanbarg, M. B., Scawthorn, C., Kiyono, J., Shahbodaghkhan, B. (2012), Fuzzy AHP-based multicriteria decision making systems using particle swarm optimization, Expert Systems vith Applications, 39 (2012), 960-966

[10] Zadeh, L. A. (1971). Similarity Relations and Fuzzy Orderings. Information Sciences 3, p. 177-200.

[11] Dukić, N. (2006), The Semantic Distance, Fuzzy Dependency and Fuzzy Formulas, Sarajevo Journal of Mathematics, Vol. 2 (15), 137-146.

[12] Zhang, X., Ma, W. and Chen, L. (2014), New Similarity of Triangular Fuzzy Number and Its Application, Hindawi Publishing Corporation, The Scientific World Journal, Volume 2014

[13] Saaty, T. L. (1980), Analytic hierarchy process, New York, McGraw-Hill. 